\title{
Editorial
}

\section{Bernhard Hennig*}

\section{International scientists seek solutions for environmental problems}

https://doi.org/10.1515/reveh-2019-0058

This Special Issue continues to advance science related to environmental challenges in Central and Eastern Europe and elsewhere, following the success of a Special Issue of Reviews on Environmental Health published in 2017 entitled "Environmental challenges in Central and Eastern Europe". Research collaborations among American and European scientists continue to address complex environmental exposures to existing or legacy pollutants, such as persistent organic pollutants, and also address challenges related to poor air quality, e-waste and other types of "modern pollution". This second Special Issue offers research articles and reviews, which not only focus primarily on environmental challenges in Central and Eastern Europe, but also draw connections to environmental health challenges that are common across many international regions. Various scientific approaches are used in different countries, but the international scientific community can advance environmental health science by sharing findings. This Special Issue exemplifies articles that highlight scientific interactions and collaborations between researchers from Central and Eastern Europe and the United States, which have resulted in scientific exchange and sharing of expertise at international conferences, such as the Central and Eastern European Conference on Health and the Environment (CEECHE).

The theme of this Special Issue is highlighted in a review by William A. Suk, which discusses advancing science in rapidly changing environments and opportunities for Central and Eastern European scientists to connect to other networks to explore challenges linked to health and disease and the environment. Suk writes, "by taking these steps to connect to the broader world of environmental health research, the Central and Eastern European region stakeholders can reduce pollution-related disease, minimize costs of hazardous waste remediation, and help grow the economy in their region". In addition to articles that are specific to the Central and Eastern European region, articles are included in this Special Issue that discuss topics that are relevant for the entire international community. Areas of emphasis in this Special Issue include (a) environmental stressors leading to respiratory symptoms in children and the assessment of child and parental reporting of symptoms relative to pollution exposure; (b) comparisons of health effects of pollutants among low-, middle- and high-income countries relative to awareness of environmental health hazards as well as better prophylaxis and medical care; (c) the importance of indoor air pollution and approaches for vapor intrusion studies, which combine building science models with vapor intrusion models; (d) studies on community resilience or the ability of a community to respond positively to adverse environmental situations; and (e) applications of metabolomics as a useful and versatile tool for characterizing the disease risks and mechanisms associated with exposure to various environmental pollutants. In summary, the peer-reviewed articles in this Special Issue span across population health impacts including environmental disease prevention strategies and community engagement when facing cumulative environmental exposure risks, as well as novel environmental monitoring and remediation technologies, linked to risk assessment and policy implications worldwide.

The Special Issue was critically peer-reviewed, and a special committee of Associate Guest Editors was appointed to delegate and assist with the review process. I wish to give special thanks to (in alphabetical order) Drs. Stephania Cormier, Stan Gawronski, Shao Lin, Slawo Lomnicki, Kelly Pennell and Michael Petriello for all their help to make the peer review process of this Special Issue a success.

Respectfully submitted, Bernhard Hennig

Guest Editor

\footnotetext{
*Corresponding author: Bernhard Hennig, Superfund Research Center, University of Kentucky, 900 S. Limestone Street, Lexington, KY 40536, USA; and Department of Animal and Food Sciences, College of Agriculture, Food and Environment, University of Kentucky, Lexington, KY 40536, USA, Phone: +1 859-218-1387, E-mail: bhennig@uky.edu
} 